\title{
Testing for Multipartite Quantum Nonlocality Using Functional Bell Inequalities
}

\author{
Q. Y. He, ${ }^{1}$ E. G. Cavalcanti, ${ }^{2}$ M. D. Reid,${ }^{1}$ and P. D. Drummond ${ }^{1}$ \\ ${ }^{1}$ Centre for Quantum-Atom Optics, Swinburne University of Technology, Melbourne, Australia \\ ${ }^{2}$ Centre for Quantum Dynamics, Griffith University, Brisbane QLD 4111, Australia
}

\begin{abstract}
We show that arbitrary functions of continuous variables, e.g. position and momentum, can be used to generate tests that distinguish quantum theory from local hidden variable theories. By optimising these functions, we obtain more robust violations of local causality than obtained previously. We analytically calculate the optimal function and include the effect of nonideal detectors and noise, revealing that optimized functional inequalities are resistant to standard forms of decoherence. These inequalities could allow a loophole-free Bell test with efficient homodyne detection.
\end{abstract}

Bell famously showed that the predictions of quantum mechanics (QM) are not always compatible with local hidden variable theories (LHV) [1]. Surprisingly, this fundamental result, which underpins the field of quantum information, has not been rigorously tested [2]. There are no experiments yet that can eliminate all LHV, either due to low detection efficiencies [3, 4] or lack of causal separation. Rigorous tests are also needed to fully implement some quantum information protocols, like that of Ekert [5] which employs a Bell inequality (BI) as a test of security in a cryptographic scheme. All of these early tests and protocols employed quantum measurements with discrete outcomes of spin or particle number.

In this Letter, we develop functional moment inequalities to test for quantum nonlocality. We then use variational calculus to optimize the choice of measured function. As a result, we obtain Bell nonlocality for larger losses and for greater degrees of decoherence than possible previously. The outcome can be feasibly tested in the laboratory, since the detectors required are efficient quadrature detectors. More generally, functional nonlocality measures could lead to new applications in quantum information. The important advantage is a much greater robustness to noise and loss.

As well as potentially overcoming the loophole problem mentioned above, formalisms to test LHV for continuous variables provide an opportunity for testing QM in new environments, and give a better understanding of the origin of the nonlocal features of QM. This is particularly true given that entanglement [6] alone does not guarantee failure of LHV for mixed states [7].

With this objective, there is the fundamental question of how to quantify the strength of nonlocality, in the absence of a single test for nonlocality that is necessary and sufficient for any quantum state. Mermin [8] used as a measure the deviation of the QM prediction from the LHV bound, based on a particular BI. A second strategy discussed recently by Cabello et al. [4] is to quantify the strength of nonlocality by the robustness of the violation with respect to a decoherence parameter. In this approach one determines the critical efficiency $\eta$ or the critical degree of purity $p$ required for a violation. Here, we evaluate all three measures to show strong correlations between them.

Recently, Cavalcanti et al. (CFRD) showed [9] that Bell inequalities can be derived for the case of observables with continuous and unbounded outcomes, like position and momentum. This approach is significant in establishing that quantum nonlocality does not rely on the discreteness of the measurement outcomes. Continuous variable (cv) inequalities also provide an avenue to understanding how manifestations of quantum nonlocality can be manipulated by choice of observable.

The original CFRD inequality [9] is $\mid\left\langle\prod_{k=1}^{N}\left(x_{k}+\right.\right.$ $\left.\left.i p_{k}\right)\right\rangle\left.\right|^{2} \leq\left\langle\Pi_{k=1}^{N}\left(x_{k}^{2}+p_{k}^{2}\right)\right\rangle$, where $x_{k}, p_{k}$ are the outcomes of two arbitrary measurements, represented in QM by observables $\hat{X}_{k}, \hat{P}_{k}$, at site $k[10]$. Where $\hat{X}$ and $\hat{P}$ are quadrature measurements with canonical position and momentum commutation relations, CFRD showed that the symmetric state $\left\{|0\rangle^{\otimes N / 2}|1\rangle^{\otimes N / 2}+\right.$ $\left.|1\rangle^{\otimes N / 2}|0\rangle^{\otimes N / 2}\right\} / \sqrt{2}$ violates the inequality for $N \geq 10$. In this case, the states $|0\rangle,|1\rangle$ are eigenstates of $a^{\dagger} a$ where $\hat{a}=\hat{X}+i \hat{P}$, so the prediction could in principle be tested with photonic Greenberger-Horne-Zeilinger (GHZ) states produced in the laboratory [11]. Note that in the above state there are $N$ field modes but only $N / 2$ photons. It can be prepared from a $N / 2$-photon $\mathrm{GHZ}$ state $\left\{|H\rangle^{\otimes N / 2}+|V\rangle^{\otimes N / 2}\right\} / \sqrt{2}$, where $|H\rangle,|V\rangle$ represent horizontally or vertically polarized single-photon states, by passing each photon through a polarizing beam splitter. These violations are robust with loss. The critical efficiency $\eta_{\text {crit }}$ required for violation tends to $\eta_{\text {crit }} \rightarrow 0.81$, as $N \rightarrow \infty$. Quadrature measurements with local oscillators are highly efficient, with reported efficiencies of 99\%. However, generation losses from mode-matching can degrade the experimental efficiency, so $81 \%$ is still a challenging practical benchmark.

Instead, we introduce a functional moment Bell inequality by considering arbitrary functions of the outcomes at each site. This new approach to nonlocality utilizes a general functional optimization of continuous variable observables. We find the optimal function that maximizes a violation of the inequality for a given effi- 
ciency $\eta$ and state purity $p$. We show that the optimal function has the form $x /\left(1+\varepsilon_{N} x^{2}\right)$, where $\varepsilon_{N}$ is a parameter related to $N$ and $\eta$. This gives an inequality which is violated by the GHZ states of (6) for $N \geq 5$. The violation increases exponentially with $N$, while $\eta_{\text {crit }}$ decreases asymptotically to 0.69 for a pure state (with $p=1$ ), thus dramatically reducing both the number of modes required, and the required efficiency.

When the functions correspond to a simple binning of a cv observable to give binary outcomes [12], our inequalities reduce to those of Mermin [8]. We extend the analysis of Mermin and Acin et al. [13], and calculate results for homodyne detection for more feasible types of state. We find that $\left(\eta p^{2}\right)_{c r i t}=2^{(1-2 N) / N} \pi$, which gives a critical efficiency for a pure state at large $N$ of $\eta=0.79$.

Functional Moment Inequalities. We present a proof of the functional moment inequality taking explicit account of functions of measurements that can be made at each of $N$ spatially separated sites. We denote the measurement made on the system at the $k$-th site by $X_{k}^{\theta}$, and the outcome of the measurement by $x_{k}^{\theta}$, where $\theta$ represents a choice of measurement parameter. Bell's assumption that LHV can describe the outcomes implies that the measurable moments $\left\langle x_{1}^{\theta} x_{2}^{\phi} \ldots x_{N}^{\varphi}\right\rangle$ can be expressed in terms of a set of hidden variables $\lambda$ as

$$
\left\langle x_{1}^{\theta} x_{2}^{\phi} \ldots x_{N}^{\varphi}\right\rangle=\int_{\lambda} d \lambda P(\lambda)\left\langle x_{1}^{\theta}\right\rangle_{\lambda}\left\langle x_{2}^{\phi}\right\rangle_{\lambda} \ldots\left\langle x_{N}^{\varphi}\right\rangle_{\lambda},
$$

where $\left\langle x_{k}^{\theta}\right\rangle_{\lambda}$ is the average of $x_{k}^{\theta}$ given a LHV state $\lambda$. Next we construct, for each site $k$, real functions of two observables $f_{k}\left(x_{k}^{\theta}\right), g_{k}\left(x_{k}^{\theta^{\prime}}\right)$, and define the complex function: $F_{k}=f_{k}\left(x_{k}^{\theta}\right)+i g_{k}\left(x_{k}^{\theta^{\prime}}\right)$. The complex moment $\left\langle F_{1} F_{2} \ldots F_{N}\right\rangle$ can be expressed in terms of realvalued expressions of the type $\left\langle f_{1}\left(x_{1}^{\theta}\right) g_{2}\left(x_{2}^{\phi^{\prime}}\right) \ldots f_{N}\left(x_{N}^{\varphi}\right)\right\rangle$, etc. Of course, $f_{k}\left(x_{k}^{\theta}\right)$ is an observable obtained from $x_{k}^{\theta}$ by local post-measurement processing. Eq. (1) must therefore also be valid for $\left\langle f\left(x_{1}^{\theta}\right) f\left(x_{2}^{\phi}\right) \ldots f\left(x_{N}^{\varphi}\right)\right\rangle=$ $\int_{\lambda} d \lambda P(\lambda)\left\langle f\left(x_{1}^{\theta}\right)\right\rangle_{\lambda}\left\langle f\left(x_{2}^{\phi}\right)\right\rangle_{\lambda} \ldots\left\langle f\left(x_{N}^{\varphi}\right)\right\rangle_{\lambda}$. For an LHV, the expectation value of products of the $F_{k}$ must satisfy:

$$
\left\langle F_{1} \ldots F_{N}\right\rangle=\int_{\lambda} d \lambda P(\lambda)\left\langle F_{1}\right\rangle_{\lambda} \ldots\left\langle F_{N}\right\rangle_{\lambda}
$$

where $\left\langle F_{k}\right\rangle_{\lambda} \equiv\left\langle f_{k}\left(x_{k}^{\theta}\right)\right\rangle_{\lambda}+i\left\langle g_{k}\left(x_{k}^{\theta^{\prime}}\right)\right\rangle_{\lambda}$. From (21), the following inequality must therefore hold:

$$
\left|\left\langle F_{1} F_{2} \ldots F_{N}\right\rangle\right|^{2} \leq \int d \lambda P(\lambda)\left|\left\langle F_{1}\right\rangle_{\lambda}\right|^{2} \ldots\left|\left\langle F_{N}\right\rangle_{\lambda}\right|^{2} .
$$

Now for any particular value of $\lambda$, the statistics predicted for $f_{k}\left(x_{k}\right)$ must have a non-negative variance, i.e., $\left\langle f_{k}\left(x_{k}\right)\right\rangle_{\lambda}^{2} \leq\left\langle f_{k}\left(x_{k}\right)^{2}\right\rangle_{\lambda}$. Writing (3) explicitly in terms of the $f_{k}$ 's and using this variance inequality we arrive at the CFRD inequality with functional moments:

$$
\left|\left\langle\prod_{k=1}^{N}\left[f_{k}\left(x_{k}^{\theta}\right)+i g_{k}\left(x_{k}^{\theta^{\prime}}\right)\right]\right\rangle\right|^{2} \leq\left\langle\prod_{k=1}^{N}\left[f_{k}\left(x_{k}^{\theta}\right)^{2}+g_{k}\left(x_{k}^{\theta^{\prime}}\right)^{2}\right]\right\rangle .
$$

We will measure the violation of this inequality by the ratio of the left- $(L H S)$ and right-hand sides $(R H S)$. Defining the Bell observable $B=L H S / R H S$, failure of $\mathrm{LHV}$ is demonstrated when $B>1$. In order to get stronger violation of local causality, we optimize the function of observables by considering

$$
\frac{\delta B}{\delta f_{k}\left(g_{k}\right)}=0
$$

Here, we consider the class of entangled states

$$
|\psi\rangle=\left(|0\rangle^{\otimes r}|1\rangle^{\otimes(N-r)}+|1\rangle^{\otimes r}|0\rangle^{\otimes(N-r)}\right) / \sqrt{2} .
$$

Thus $r=N$ corresponds to extreme photon-numbercorrelated states, a superposition of a state with 0 photons at all sites and a state with 1 photon at each site. Next, we consider how to optimize the function $f_{k}$ and $g_{k}$ to generate a robustly violated inequality, including losses and noise.

We use variational calculus to find the optimal function using the condition of Eq. (5). For simplicity, we assume the functions $f_{k}$ and $g_{k}$ are odd. The $L H S$ can be maximized by choosing orthogonal angles, while the $R H S$ is invariant with angles. We find that

$$
B_{N}=\frac{2^{N-1}\left(\frac{2}{\pi}\right)^{\frac{N}{2}}\left(\prod_{k=1}^{N} I_{k}^{+}+\prod_{k=1}^{N} I_{k}^{-}\right)^{2}}{\prod_{k=1}^{r} I_{k} \prod_{k=r+1}^{N} I_{k}^{0}+\prod_{k=1}^{r} I_{k}^{0} \prod_{k=r+1}^{N} I_{k}}
$$

where $I_{k}^{ \pm}=2 \int e^{-2 x^{2}} x f_{k}^{ \pm} d x, I_{k}=4 \int x^{2} e^{-2 x^{2}}\left[\left(f_{k}^{+}\right)^{2}+\right.$ $\left.\left.\left(f_{k}^{-}\right)^{2}\right)\right] d x$, and $\left.I_{k}^{0}=\int e^{-2 x^{2}}\left[\left(f_{k}^{+}\right)^{2}+\left(f_{k}^{-}\right)^{2}\right)\right] d x$ are different integrals for $x$ which contribute to the expectation values in both sides of inequality (4). Here $f_{k}^{ \pm}=f_{k} \pm g_{k}$, and the factor $e^{-2 x^{2}}$ was obtained from the joint probability of observables. Requiring $\delta B_{N} / \delta f_{k}^{ \pm}=0$, we find the optimal condition: $f_{k}(x)= \pm g_{k}(x)$. The components of complex functions $f_{k}, g_{k}$ are the same at each site, and have the form

$$
f_{k}(x)=g_{k}(x)=\frac{x}{1+\varepsilon_{N} x^{2}}
$$

For the even $N$ case, it is optimal to choose $r=N / 2$. Then $\varepsilon_{N}$ is independent of $N$, but has to be calculated numerically since it satisfies a nonlinear integral equation: $\varepsilon_{N}=4 I^{0} / I$.

For $N$ an odd number, the greatest violations occur for $r=(N-1) / 2$. The optimal function has the same form as in (8) except that the parameter $\varepsilon_{N}$ changes to $\varepsilon_{N}^{\prime}$, where:

$$
\varepsilon^{\prime}{ }_{N} \equiv \varepsilon_{N}\left[\frac{N \varepsilon_{N}^{+}-\varepsilon_{N}^{-}}{N \varepsilon_{N}^{+}+\varepsilon_{N}^{-}}\right]
$$

and $\varepsilon_{N}^{ \pm}=\varepsilon_{N} \pm 4$. However, the numerical value of $\varepsilon_{N}$ and $\varepsilon_{N}^{\prime}$ now depend on $N$, as the integral equation (9) 
for odd values of $N$ is $N$-dependent. This provides better violations of (4) than any other arbitrary function, provided $N \geq 5$. The maximum $B_{N}$ value with this optimal choice is shown in Fig. 1, compared with the CFRD result which uses a simple correlation function.

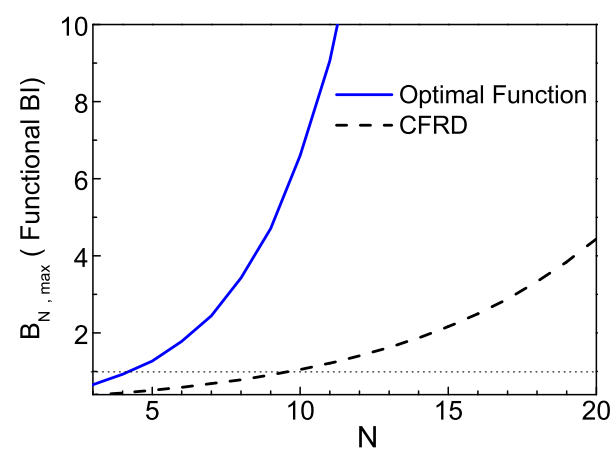

Figure 1: Maximum violations of functional cv inequality with GHZ states as a function of the number of modes. The violations using the optimal function (solid) are much stronger than the CFRD result (dashed).

Binned cv outcomes for Mermin-Klyshko inequality (MK) [14]. We will also briefly consider binning methods. Specifically, we define the binning functions $f_{k}(x)=$ $g_{k}(x)=f_{b i n}(x)=+1$ if $x \geq 0$ and -1 otherwise. For such discrete outcomes, the original formalisms of Mermin and Klyshko [8, 14] can be used. The CFRD inequality for discrete outcomes reduces to that of Mermin [8], as can be seen by noting that $\left[f_{b i n}\right]^{2}=1$. Here it is known that the Bell inequality introduced for this discrete case by Klyshko is stronger. Defining $F_{k}=$ $f_{\text {bin }}\left(x_{k}^{\theta}\right)+i f_{\text {bin }}\left(x_{k}^{\theta^{\prime}}\right)$ and $\Pi_{N}=\prod_{k=1}^{N} F_{k}$, we can use the MK inequality $\left|S_{N}\right| \leq 1$, where $S_{N}=2^{-N / 2}\left[\operatorname{Re}\left\{\Pi_{N}\right\} \pm\right.$ $\left.\operatorname{Im}\left\{\Pi_{N}\right\}\right]$, for $N$ even, and $S_{N}=2^{-(N-1) / 2} \operatorname{Re}(\operatorname{Im})\left\{\Pi_{N}\right\}$ or $S_{N}=2^{-(N-1) / 2} \sqrt{\left(\operatorname{Re}\left\{\Pi_{N}\right\}\right)^{2}+\left(\operatorname{Im}\left\{\Pi_{N}\right\}\right)^{2}}$ for $N$ odd. These BI have been considered recently for the case of extreme photon-number correlated states, where $r=N$, by Acin et al. We can also define $\Pi_{N}$ by exchanging the local observables, to obtain similar inequalities.

We generalize this approach to account for more general angles and states. We find an optimal violation of $B_{N}=\frac{\sqrt{2}}{2}\left(\frac{4}{\pi}\right)^{N / 2}$, for arbitrary $r$ and $N$, with the optimal phases: $\theta_{k}=(-1)^{N+1} \pi(k-1) /(2 N), \theta_{k}^{\prime}=\theta_{k}+\pi / 2$ for $k \leq r$, and $\theta_{k}=(-1)^{N} \pi(k-1) /(2 N), \theta_{k}^{\prime}=\theta_{k}-\pi / 2$ for $k>r$. This result has been presented by Acin et al. [13] for the special case of $r=N$. We confirm the exponential increase with number of sites $N$, but also make the observation that the violation occurs for all types of states of the form (6), independently of $r$. This contrasts with the result for the CRFD inequality, which requires $r \sim N / 2$ for violation. However, as explained earlier, the states with $r=N / 2$ are straightforwardly feasible given a polarisation GHZ state, as opposed to the extreme photon-number correlated states considered by Acin et al. Therefore, this is a very important experimental advantage. Violation of the MK inequality with binning is possible for $N \geq 3$, but, as we will see, this strategy is sensitive to losses and noise.

Sensitivity to loss and state impurity. The value of the Bell observable $B_{N}$ increases with the number of sites $N$, so this is suggestive of a strategy that will allow genuine loophole-free violations of local causality. However, it may be argued that since increasing the number of sites will increase the number of detectors required, there will be no advantage. Only careful calculation of the Bell observable $B_{N}$ including the detection efficiency $\eta$ can determine whether the strategy is advantageous.

Loss is modeled as follows. The field modes $a_{k}$ at each site are independently coupled to a second mode $a_{k, v a c}$ respectively, assumed to be in a vacuum. Photons are lost from the field into the vacuum mode, the strength of coupling determining the rate of loss. This beam splitter model gives the final detected and vacuum mode in terms of the inputs $a$ and $a_{v a c}$

$$
\begin{aligned}
a_{\text {out }} & =\sqrt{\eta} a+\sqrt{1-\eta} a_{v a c}, \\
a_{\text {vac }, \text { out }} & =\sqrt{1-\eta} a-\sqrt{\eta} a_{v a c},
\end{aligned}
$$

where $\eta$ is the efficiency, the probability of detecting a photon after coupling. Since we only measure the " $a_{\text {out }}$ " not the " $a_{v a c, \text { out }}$ ", we need to trace over the latter modes to obtain the final density operator $\rho_{\text {out }}$ for the detected modes after loss. We can also examine the effect of impurity, by considering a state $\rho^{\prime}=p|\psi\rangle\langle\psi|+$ $(1-p) \rho_{m i x}$, where $\rho_{m i x}$ is the mixed state obtained with a model for decoherence in the occupation-number basis, i.e. $\quad \rho_{\operatorname{mix}}=\left[|0\rangle^{\otimes r}|1\rangle^{\otimes(N-r)}\left\langle\left. 0\right|^{\otimes r}\left\langle\left. 1\right|^{\otimes(N-r)}+\right.\right.\right.$ $|1\rangle^{\otimes r}|0\rangle^{\otimes(N-r)}\left\langle\left. 1\right|^{\otimes r}\left\langle\left. 0\right|^{\otimes(N-r)}\right] / 2\right.$, and $p$ is the probability the system is in the original pure state (6).

Including the effect of detection inefficiencies and noise, the parameter $\varepsilon_{N}$ is changed to $\varepsilon_{N}(\eta)$ for the optimum function. For $N$ even we find that

$$
\begin{aligned}
\varepsilon_{N}(\eta) & =\frac{2 \eta \varepsilon_{N}}{2 \eta+(1-\eta) \varepsilon_{N}}, \\
B_{N} & =2^{N-2}\left[\frac{2\left(I^{+}\right)^{4}(\eta p)^{2}}{\pi I^{o} C}\right]^{\frac{N}{2}},
\end{aligned}
$$

where $\varepsilon_{N}$ is defined as before, and $C=\eta I+(1-\eta) I^{0}$. For the case of odd $N$ the relevant integral equations change, giving a modified (and slightly reduced) Bell variable $B_{N}^{\prime}$, where:

$$
\begin{aligned}
\varepsilon_{N}^{\prime}(\eta) & =\varepsilon_{N}(\eta) \frac{N \varepsilon_{N}^{+}(\eta)-\varepsilon_{N}(\eta) \varepsilon_{N}^{-} / \varepsilon_{N}}{N \varepsilon_{N}^{+}(\eta)+\varepsilon_{N}^{2}(\eta) \varepsilon_{N}^{-} / \varepsilon_{N}^{2}} \\
B_{N}^{\prime} & =\frac{2 \sqrt{I^{0} C}}{I^{0}+C} B_{N} .
\end{aligned}
$$

Here $\varepsilon_{N}^{+}(\eta)=\varepsilon_{N}(\eta)+4$, and $B_{N}$ is defined as in Eq (11). 

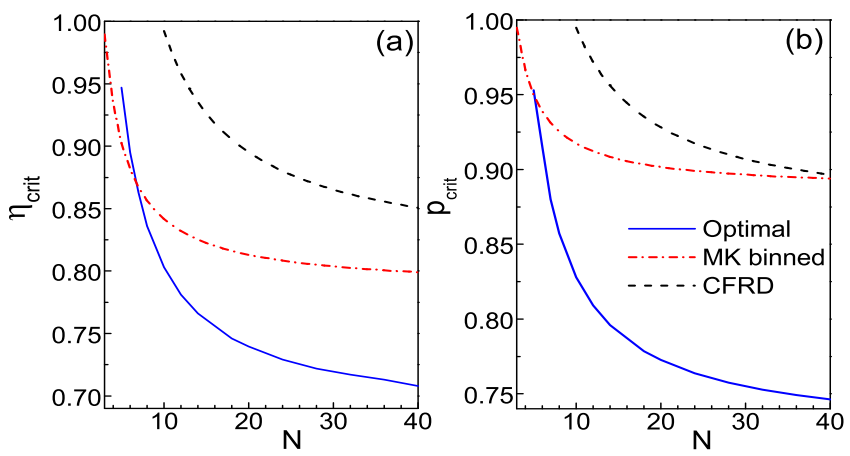

Figure 2: (a) The critical minimum detection efficiency $\eta_{\text {crit }}$ for pure state, and (b) the critical purity $p_{\text {crit }}$ for ideal detectors required for violation of functional moment, CFRD and MK inequalities with optimal choice of parameters.

This approach is applied to enable a prediction of the effect of loss and noise on the functional inequalities, and the results are plotted in Fig. 2. These results can be compared with the MK binning approach. With the choice of optimal angles, we find the values of the MK Bell observable with binned cv outcomes is $B_{N}(p, \eta)=\frac{\sqrt{2}}{2}\left(\frac{4 \eta p^{2}}{\pi}\right)^{N / 2}$, which gives the effect of detection inefficiencies and noise for the optimal choice of angles. That implies a critical minimum efficiency and purity $\left(\eta p^{2}\right)_{\text {crit }}=2^{(1-2 N) / N} \pi$ in order to violate the inequality. For lower $N$, the strategy of binning and using the MK inequality shows an advantage, by allowing a violation for $N=3,4,5$, - but even if $p=1$, high efficiencies $\eta>0.99,0.93,0.90$ are required. While high detection efficiencies are feasible for homodyne detection, these efficiency and purity values are still quite challenging once generation losses are also taken into account. In view of this, the high requirement for $\eta_{\text {crit }}$ for the case $N=3$ may be prohibitive.

These results show that the functional inequality has much greater robustness against noise and inefficiency than the MK inequality. For $N>7$, the functional $\mathrm{cv}$ inequality used with an optimal function allows violation of LHV at much lower efficiencies and larger maximum noise. The asymptotic decoherence product is $(p \eta)_{\infty} \sim 0.6918$ in the large $\mathrm{N}$ limit. For a moderate efficiency $\eta_{\text {crit }} \sim 80 \%$ one requires $N=10$ if the optimized function, while the binned MK case requires $N \sim 40$.

In conclusion, we have developed a new direction for the analysis of cv nonlocality. For the input state treated here, the optimal measured function always has the same functional form apart from changing the parameter $\epsilon$, but more generally, the functional form may depend on the experimental decoherence. Future research may include further optimization of the functions for different entan- gled states and application of this method to tests of other forms of nonlocality-i.e., entanglement 15] and EPR steering [16].

We wish to acknowledge funding for this project from the Australian Research Council through a Discovery grant and the ARC Centre of Excellence for QuantumAtom Optics. EGC acknowledges discussions with Antonio Acin, Daniel Cavalcanti and Yeong-Cherng Liang.

[1] J. S. Bell, Physics 1, 195 (1964).

[2] A. Garg and N. D. Mermin, Phys. Rev. D 35, 3831 (1987); J. A. Larsson, Phys. Rev. A 57, R3145 (1998); 59, 4801 (1999); 57, 3304 (1998); J. A. Larsson and J. Semitecolos, ibid. 63, 022117 (2001); A. Cabello and J. A. Larsson, Phys. Rev. Lett. 98, 220402 (2007).

[3] J. A. Larsson, Phys. Lett. A 256, 245 (1999); P. M. Pearle, Phys. Rev. D 2, 1418 (1970).

[4] A. Cabello, D. Rodriguez and I. Villanueva, Phys. Rev. Lett. 101, 120402 (2008).

[5] A. K. Ekert, Phys. Rev. Lett. 67, 661 (1991); V. Scarani and N. Gisin, ibid. 87117901 (2001); J. A. Larsson, Quantum Inf. Comput. 2, 434 (2002).

[6] A. Peres, Phys. Rev. Lett. 77, 1413 (1996).

[7] R. F. Werner, Phys. Rev. A 40, 4277 (1989).

[8] N. D. Mermin, Phys. Rev. Lett. 65, 1838 (1990); M. Ardehali, Phys. Rev. A 46, 5375 (1992). D. M. Greenberger, M. A. Horne, and A. Zeilinger, in Bell's Theorem, Quantum Theory, and Conceptions of the Universe, edited by M. Kafatos (Kluwer Academic, Dordrecht, Holland, 1989), p. 69.

[9] E. G. Cavalcanti, C. J. Foster, M. D. Reid, and P. D. Drummond, Phys. Rev. Lett. 99, 210405 (2007).

[10] Bearing in mind these measurements can be noncompatible, the LHS is simply an abbreviation for the expansion in terms of observable moments, e.g. $\left\{\left\langle x_{1} x_{2}\right\rangle-\right.$ $\left.\left\langle p_{1} p_{2}\right\rangle\right\}^{2}+\left\{\left\langle x_{1} p_{2}\right\rangle+\left\langle x_{2} p_{1}\right\rangle\right\}^{2}$ when $N=2$.

[11] C. A. Sackett, et al., Nature (London) 404, 256 (2000); Z. Zhao, et al., Phys. Rev. Lett. 91, 180401 (2003); Z. Zhao, et al., Nature (London) 430, 54 (2004); D. Leibfried, et al., Nature (London) 438, 639 (2005); C. Y. Lu, et al., Nature Phys. 3, 91 (2007).

[12] U. Leonhardt and J. A. Vaccaro, J. Mod Opt, 42, 939 (1995); A. Gilchrist, P. Deuar, M. D. Reid, Phys. Rev. Lett. 80, 3169 (1998); B. Yurke, M. Hillery, and D. Stoler, Phys. Rev. A 60, 3444 (1999); J. Wenger et al., ibid., 67, 012105 (2003).

[13] A. Acin, N. J. Cerf, A. Ferraro, and J. Niset, Phys. Rev. A 79, 012112 (2009).

[14] D. N. Klyshko, Phys. Lett. A 172, 399 (1993); A. V. Belinskii and D. N. Klyshko, Phys. Usp. 36, 653 (1993).

[15] V. Giovannetti, S. Mancini, D. Vitali, and P. Tombesi, Phys. Rev. A 67, 022320 (2003); M. Hillery and M. S. Zubairy, Phys. Rev. Lett. 96, 050503 (2006).

[16] E. G. Cavalcanti, S. J. Jones, H. M. Wiseman and M. D. Reid, Phys. Rev. A, 80, 032112 (2009). 